\title{
USE OF ARTIFICIAL NEURAL NETWORKS FOR FUSION OF INFRARED AND VISION SENSORS IN A MOBILE ROBOT NAVIGATION SYSTEM
}

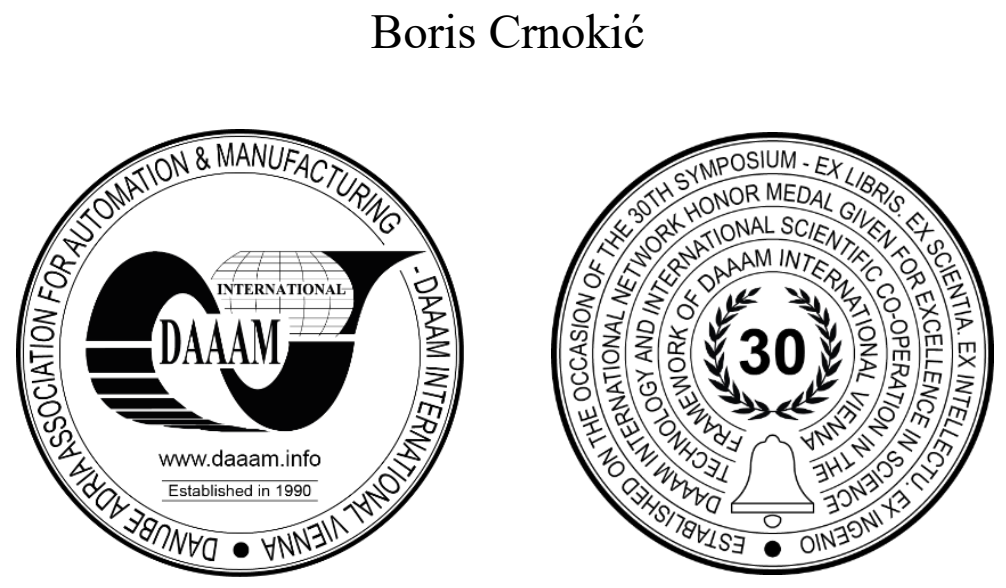

This Publication has to be referred as: Crnokic, B[oris] (2020). Use of Artifical Neural Networks for Fusion of Infrared and Vision Sensors in a Mobile Robot Navigation System, Proceedings of the 31st DAAAM International Symposium, pp.0080-0087, B. Katalinic (Ed.), Published by DAAAM International, ISBN 978-3-902734-29-7, ISSN 1726-9679, Vienna, Austria

DOI: $10.2507 / 31$ st.daaam.proceedings.011

\begin{abstract}
This paper presents a mobile robot navigation system that uses artificial neural networks for sensor fusion. The control system is based on the fusion of data from three infrared sensors and one vision sensor (VGA camera). Data from VGA camera was used to detect edges of obstacles in the environment, while infrared sensors were used to measure the distance from obstacles. Canny edge detector and LPQ descriptor were used for image pre-processing, i.e. edge detection and extraction of features from images. A multilayer perceptron network trained by the backpropagation algorithm was used to classify the detected obstacles. The control algorithm was implemented in the MATLAB software package and tested on the Robotino 2 mobile robot. The experiment was done indoors with static obstacles that represent the real environment. This experiment showed that the developed algorithm gives very good results with an accuracy of $72.07 \%$. Ultimately, navigation system performs the tasks of detecting and avoiding most of the obstacles on which it was tested.
\end{abstract}

Keywords: Mobile robot navigation; Sensor fusion; Infrared sensors; Camera; Artificial neural networks

\section{Introduction}

The problem of mobile robot navigation is one of the key factors in the design and development of intelligent mobile robots. There are different navigation systems which are based on one or more types of sensors. One of the key problems in the application of all these systems are the tasks of robot self-location, route planning and construction of environmental maps. The solution to these problems is to combine several different types of sensors, i.e. to make a fusion of data (measurements) from several different sensors. In the field of sensor fusion in general, and therefore in the field of sensor fusion for navigation of mobile robots, there are many different approaches and solutions.[1]

Through an overview of previous work in this field, some of the fusion systems with different types of sensors are presented, and each of them is designed to create the most efficient and accurate navigation system of mobile robots. There are many sensor fusion methods used in mobile robotics, such as: Bayesian Decision Theory, Dempster-Shafer's (DS) evidence theory, weight factor model, Kalman filter, fuzzy logic, artificial neural networks, etc. 
[2]-[4] Artificial neural networks, and their application, have received special attention in recent decades in many fields such as information processing and control systems, which includes the navigation of mobile robots. [5] Supporting vector machines (SVMs) were used to extract numbers from images in the navigation system, where chair numbers were used as landmarks to localize the mobile robot. [6] Many data fusion approaches are based on the Principal Component Analysis (PCA) method, such as robot position estimation based on the analysis of principal data components from a laser distance sensor. [7] Another approach to sensor fusion, in solving mobile robot navigation problems, is the Sequential Principal Components (SPC) method inspired by Hebbian neural network learning theory. [8] One new concept, selfadaptive simulated human intelligence algorithm, is shown through the fusion of ultrasonic and infrared sensors based on intelligent artificial neural network technologies. [9] Another system of fusion of infrared and ultrasonic sensors was realized by using Back Propagation (BP) neural network algorithm in the examples of obstacles avoidance, for the real case of an intelligent mobile robot navigation. [10] In paper [11], a multilayer model of a neural network for avoiding obstacles through enhanced learning is presented. An feed-forward neural network with a multilayer perceptron was applied to different cases: to correct nonlinear errors of distance data from infrared sensors during the construction of the mobile robot environment map [12], in the process of camera calibration for mobile robot global localization by using a monocular camera [13], to translate sonar readings into occupancy values for each of the cells of the metric map construction network [14], for data fusion from infrared sensors and sonar [15], for fusion of information obtained from camera, in the process of image processing and measuring the distance from infrared sensors [16]. The development of a vision system based on an artificial neural network for autonomous movement of a mobile robot is presented in paper [17].

The navigation system presented in this paper was tested and applied on the Robotino 2 mobile robot. The experiment was realized in two parts, through simulation in the Robotino SIM software environment [18] and through the application of the algorithm in a real environment. The control algorithm is implemented in the MATLAB software package. Fig. 1. shows a mobile robot Robotino 2, an IR sensors layout, and a camera.

\section{Hardware and software used}

Hardware used in this experiment includes mobile robot Robotino 2 (Figure 1.) and mobile PC (laptop), while the navigation system algorithm is implemented in programming environment MATLAB/Simulink. Programming environment RobotinoView 2 was used to capture and save images from the environment and to manually control the robot. Fig. 1. shows a mobile robot Robotino 2, an IR sensors layout, and a VGA camera.

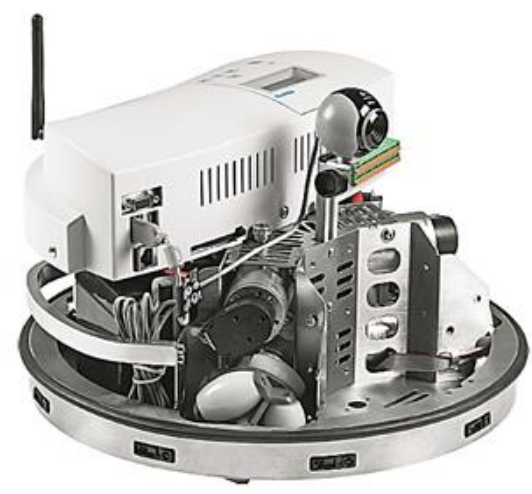

Robotino

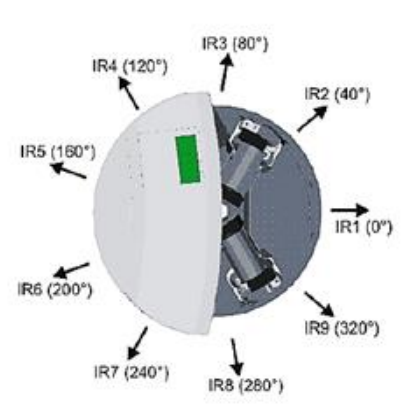

IR sensor layout

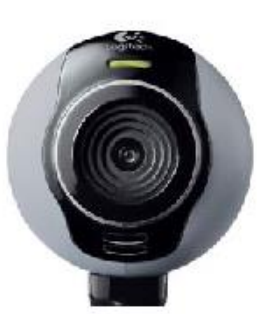

Camera

Fig. 1. Robotino with sensors

Robotino 2 possesses various types of sensors, actuators and software interfaces. Some of these sensors come as standard equipment, such as: 9 infrared sensors (IR sensors), VGA camera, incremental encoder and anti-collision sensor. In our case, the Logitec C250 camera was used to collect data (images and video) from the environment, whose height and inclination can be regulated. Three infrared sensors SHARP GP2D120 (IR1, IR2 and IR9) were used to measure the distance from the obstacles in front of the robot. Infrared sensors have the ability to detect objects at a distance from 3 to $40 \mathrm{~cm}$. All 9 infrared sensors (IR1 - IR9) on robot are arranged on a chassis at an angle of $40^{\circ}$. Robotino moves with three independent, omnidirectional drive units, mounted at an angle of $120^{\circ}$ relative to another drive. This drive system enables movement in all directions: forward, backward, sideways and rotation. The system can work independently as well as linked to the external computer via Wi-Fi connection.

\section{Proposed control algorithm}

Considering some of the systems in the mentioned papers in the introductory chapter ([5], [9], [10], [12], [16], [17]), the structure of the proposed control algorithm is shown in Figure 2. 


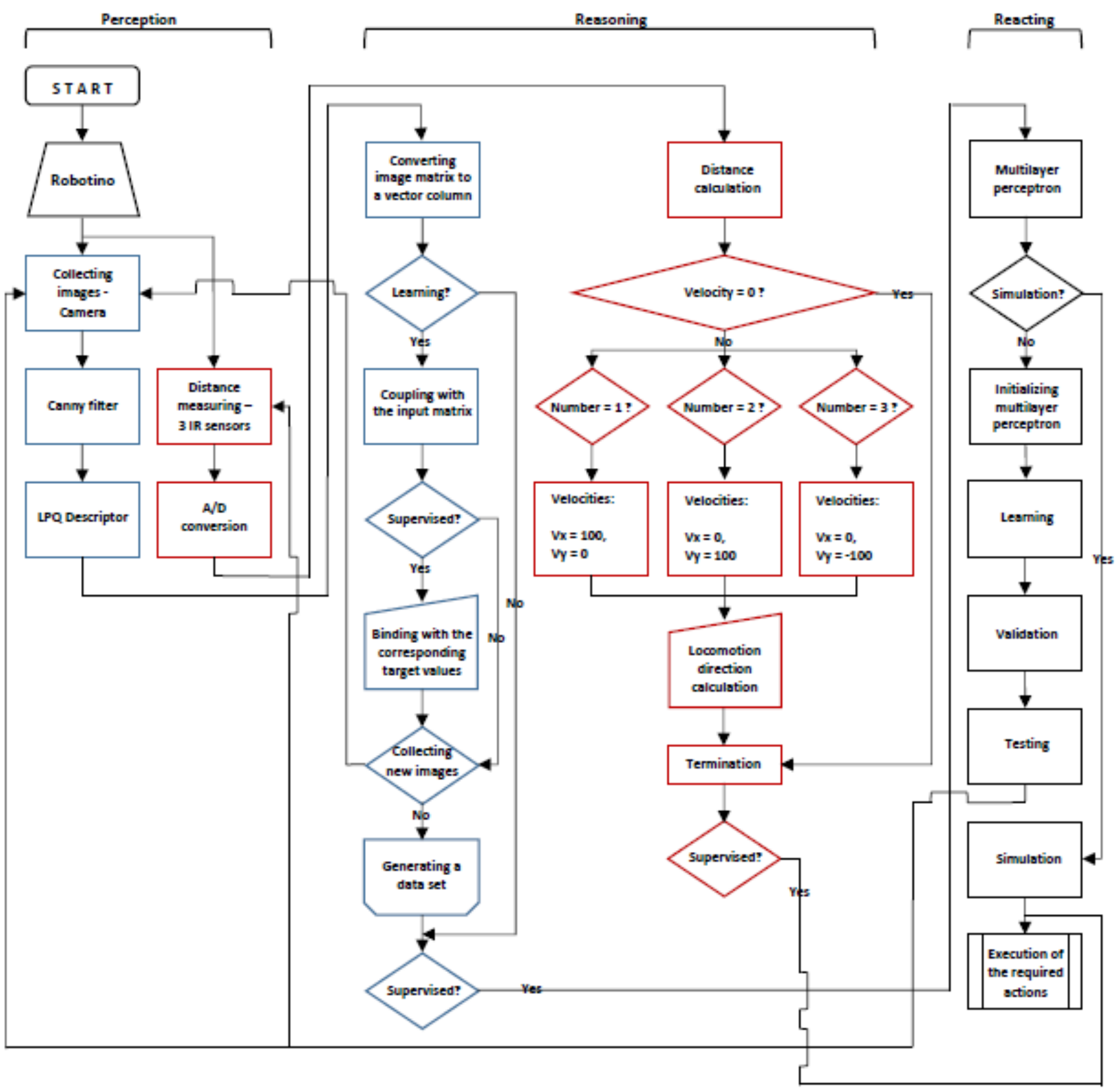

Fig. 2. Proposed control algorithm

The structure of the algorithm is divided into three segments: Perception, Reasoning, and Reacting. In the "Perception" part, data is collected and pre-processed from three infrared sensors and a camera. Through the "Reasoning" part, the system processes the data received from the sensor, compares it with the reference required input values, and makes a judgment for the robot's output movements. Finally, in the "Reacting" part, the algorithm provides the final calculations to take the necessary actions such as robot movement or eventual avoidance of obstacles.

Infrared sensors (IR1 - IR9) and cameras installed on Robotino are used to provide information about robot's environment. In order to simplify the system and to reduce the computational complexity of the control algorithm, in the experiment only three front IR sensors are used, labelled as IR1, IR2 and IR9. Using only these three sensor proved to be sufficient to detect obstacles in front Robotino, or left and right of the Robotino. Obstacles detection is organized in two parts, separate for readings from the VGA camera and from three IR sensors. The camera serves to detect landmarks and for localization, i.e. to detect edges, overhangs, and free space for unobstructed locomotion of the robot. IR sensors are used to measure distance from obstacles and to detect additional obstacles in free space in real time. Depending on whether one of the three IR sensors has detected an obstacle, the obstacle avoidance vector is determined, i.e. the velocities $\mathrm{v}_{\mathrm{x}}$ in the direction of the robot movement along the $\mathrm{x}$ axis and $\mathrm{v}_{\mathrm{y}}$ in the direction of the robot movement along the $\mathrm{y}$ axis are set.

For image pre-processing, i.e. for detection of edges and extraction of features Canny method is used combined with LPQ descriptor. Multilayer perceptron network performs the task of training the system for detecting obstacles in the environment, using data obtained from the VGA camera. 
In order to reduce the computational complexity of the control algorithm, only certain obstacle samples were taken to train the neural network. After training the network, the system is able to decide whether the robot should continue to move forward, if no trace of obstacles is detected, or to avoid an obstacle if any of them is detected. Additional information on detected obstacles are coming from 3 IR sensors. These data sets merge with the previous data gathered from the VGA camera to give the final confirmation to the robot, which direction of motion should be selected. [18]

\section{Experiment setup}

The control algorithm was tested indoors with real obstacles. To train the neural network in this experiment, 36 images of obstacles were taken. The artificial neural network in this experiment has one input, one output and one hidden layer. The input layer consists of 256 nodes representing the image obtained after pre-processing. These inputs are used as a 256-dimensional vector of image features, formed from the histogram of the integer values of all image positions. In fact, it presents 256 hypercaps in eight-dimensional space, which gives an 8-bit code. Since 36 pictures were taken for training, the network input is essentially a 256x36 matrix. The hidden layer has 10 nodes. The output layer has 3 nodes, where nodes represent outputs that determine one of the three input image classes, and finally determine the direction of movement of the robot. The structure of the artificial neural network is shown in Figure 3.

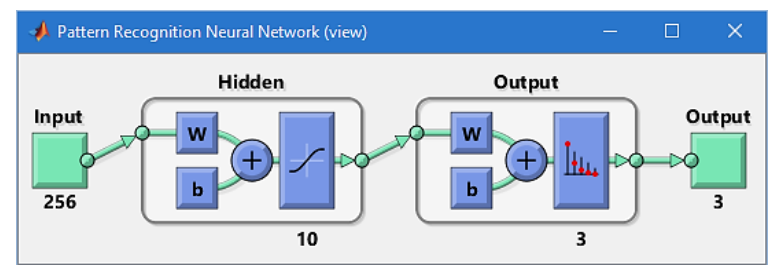

Fig. 3. Structure of the artificial neural network

Obstacles are divided into three classes. All images were collected under different room lighting conditions, and from different obstacle approach positions. The first class includes obstacles shown in Figure 4, under numbers 1-12. Obstacles are essentially part of the table, i.e. obstacles are table legs and cross girders (overhangs). In the second class of obstacles there are three boxes of different shapes, dimensions and colours, shown in Figure 4 under numbers 13-24. The third class of obstacles is shown in Figure 4, under numbers 25-36. This class is formed from the edges of the wooden table on which the Robotino is placed. In addition to the already mentioned obstacles from three classes, one obstacle was taken for testing the algorithm.

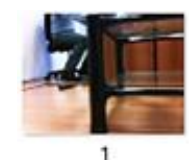

1

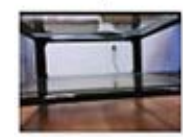

7

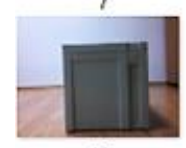

13

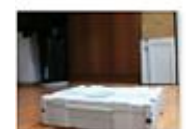

19

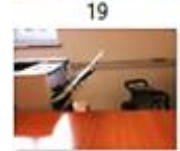

25

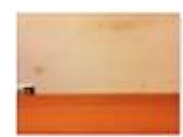

31

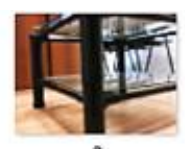

2

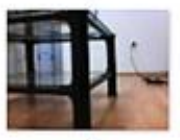

8

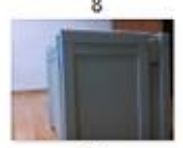

14

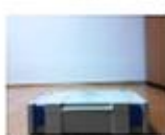

20

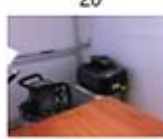

26

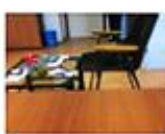

32

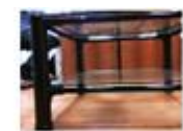

3

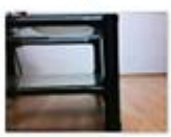

9

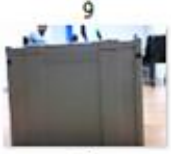

15

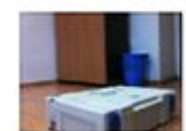

21

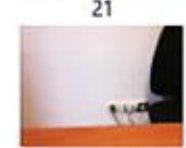

27

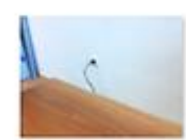

33

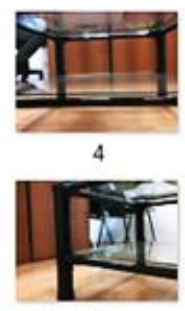

10

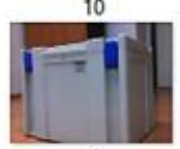

16

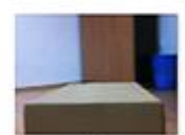

22

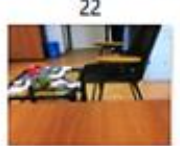

28

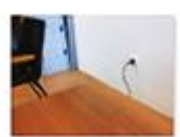

34

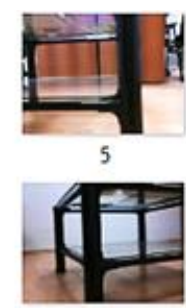

11

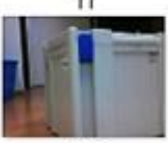

17

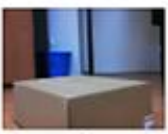

23

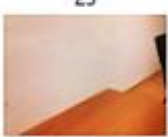

29

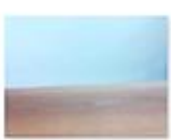

35

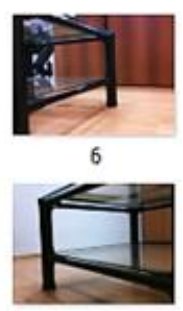

12

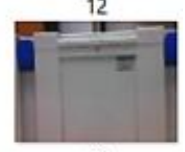

18

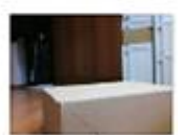

24

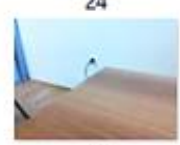

30

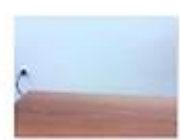

36

Fig. 4. Obstacles used in the experiment 
In order to collect data for proof of the efficiency of the proposed algorithm, obstacles were classified according to the type of obstacle, the type of sensor that detected the obstacle and the accuracy of detecting a particular obstacle, as shown in Table 1. For the purposes of this experiment 6 obstacles were taken. The table with the results of the experiment is formed as follows: the number of attempts to detect each obstacle is 20, all successful and unsuccessful attempts are registered, the type of sensor that detected the obstacle for a particular attempt is also registered.

The table shows the following obstacles: $O b 1$ - table with overhangs/1st class of obstacles; $O b$ 2, Ob 3,Ob $4-$ boxes/2nd class of obstacles; $O b 5$ - table edges/3rd class obstacle; $O b 6$ - walls/obstacles for testing. Mobile robot approached the obstacles from different sides, at different approach angles and from different distances. Successful obstacle detection is marked with "YES", while failed obstacle detection is marked with "NO". Successful detection is further classified according to the type of sensor (VGA camera - "Cam", infrared sensors - "IR") that detected a particular obstacle. With regard to detected and undetected obstacles, the accuracy of obstacles detection and the share of individual sensors in detecting of obstacles were determined. Obstacle avoidance is done in the manner shown in Figure 5.

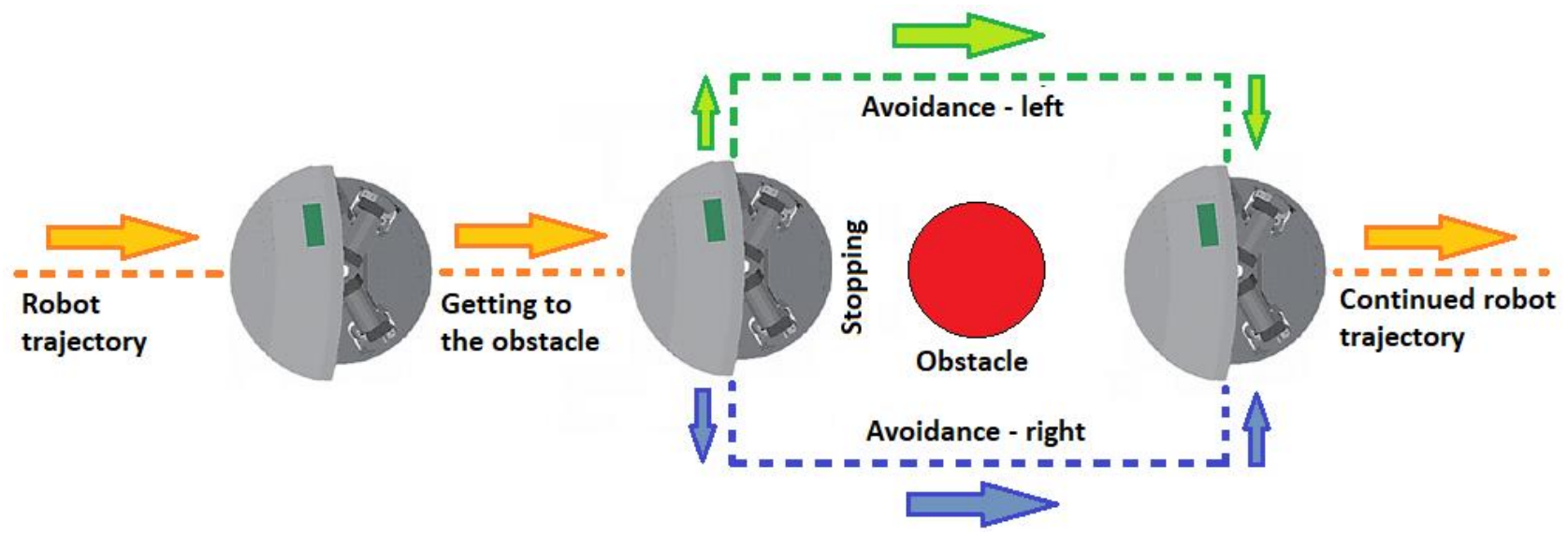

Fig. 5. Avoidance of obstacles

First, the Robot moves in a straight line. After encountering an obstacle and detecting it, it stops short to calculate and to make a decision how to avoid an obstacle. After detecting an obstacle, the robot has two solutions to avoid the obstacle: by a path to the left or another path to the right. After avoiding the obstacle, the robot returns to the same straight path as it was before encountering the obstacle.

\section{Overview and analysis of experimental results}

Table 1. shows all attempts to detect different obstacles:

\begin{tabular}{|c|c|c|c|c|c|c|c|c|}
\hline $\begin{array}{c}\text { Obstacle } \\
\text { type }\end{array}$ & \multicolumn{3}{|c|}{ Detection } & \multicolumn{2}{c|}{ Sensor type } & \multicolumn{2}{c|}{ Sensor (\%) } & $\begin{array}{c}\text { Accuracy } \\
(\%)\end{array}$ \\
\hline Yb 1 & 18 & 2 & 20 & 10 & 8 & 55,56 & 44,44 & $\mathbf{9 0}$ \\
Ob 2 & 20 & 0 & 20 & 3 & 17 & 15,00 & 85,00 & $\mathbf{1 0 0}$ \\
Ob 3 & 20 & 0 & 20 & 1 & 19 & 5,00 & 95,00 & $\mathbf{1 0 0}$ \\
Ob 4 & 20 & 0 & 20 & 3 & 17 & 15,00 & 85,00 & $\mathbf{1 0 0}$ \\
Ob 5 & 13 & 7 & 20 & 13 & 0 & 100 & 0 & $\mathbf{6 5}$ \\
Ob 6 & 20 & 0 & 20 & 2 & 18 & 10,00 & 90,00 & $\mathbf{1 0 0}$ \\
\hline \hline$\sum$ & $\mathbf{1 1 1}$ & $\mathbf{9}$ & $\mathbf{1 2 0}$ & $\mathbf{3 2}$ & $\mathbf{7 9}$ & $\mathbf{2 8 , 8 3}$ & $\mathbf{7 1 , 1 7}$ & $\mathbf{9 2 , 5 0}$ \\
\hline
\end{tabular}

Table 1. Classification of obstacle detection accuracy

Total number of attempts to detect all obstacles is 120 . Of these 120 attempts, 111 were successful, while in 9 cases the detection of obstacles was not successful. This ratio of detected and undetected obstacles results in the accuracy of obstacles detection of $92.50 \%$. Obstacles that had the highest percentage of detection are obstacles of similar geometric characteristics, such as boxes and walls. One of the problems related with obstacles detection in a real environment was noticed during the processing of data when collecting images from the camera, which resulted in certain interference in the flow of information in real time. Three infrared sensors detected obstacles 79 times, while the camera detected some of the obstacles 32 times. IR sensors detected boxes and walls better, while the camera had more detections with overhangs. 
The camera was used entirely for detecting the edges of the table, i.e. all 13 detections were performed with VGA camera. The Ob 6 obstacle (walls) is not included in the artificial neural network training set, however this obstacle has $100 \%$ accurate detections with a higher percentage of IR sensor detection.

The following limitations should be considered when avoiding detected obstacles in a real environment: only three IR sensors are used, three IR sensors cover only an angle of $80^{\circ}$ in front of the robot, camera immobility problem, robot movement only in directions: forward, left, right, the inability to rotate the robot around its axis and backward movement, processing time of detected obstacle data. These restrictions have made it impossible to avoid some obstacles, resulting in collisions with certain obstacles. As an indicator of the impact of the aforementioned limitations, Figure 6 shows the ratio of collisions compared to the number of detected and avoided obstacles.

The ratio of avoided and detected obstacles in relation to collisions with other obstacles

Ob 6

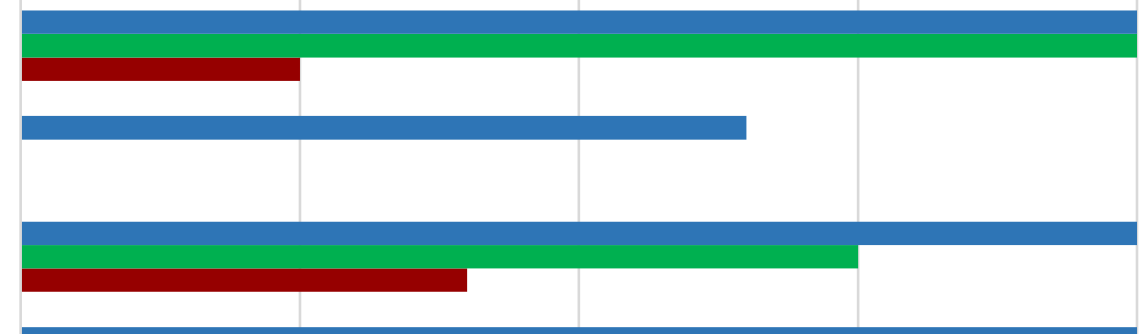

Ob 3

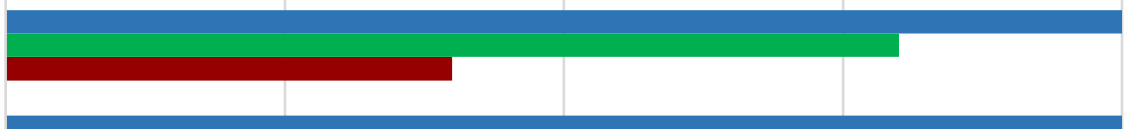

Ob 2

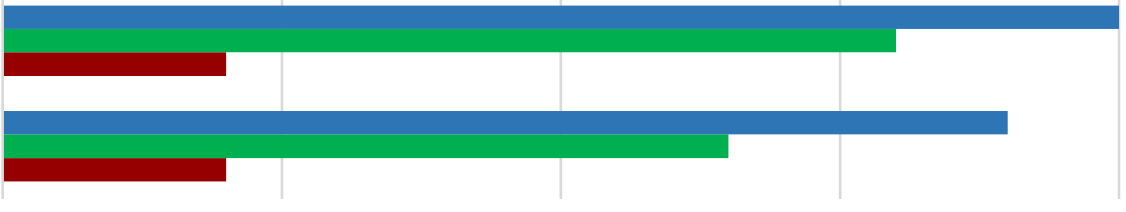

0

5

10

15

20

25

\begin{tabular}{|l|c|c|c|c|c|c|}
\hline & Ob 1 & Ob 2 & Ob 3 & Ob 4 & Ob 5 & Ob 6 \\
\hline Detected & 18 & 20 & 20 & 20 & 13 & 20 \\
\hline - Avoided & 13 & 16 & 16 & 15 & 0 & 20 \\
\hline - Colisions & 4 & 4 & 8 & 8 & 0 & 5 \\
\hline
\end{tabular}

Fig. 6. The ratio of avoided and detected obstacles in relation to collisions with other obstacles

Table 2. shows the accuracy of the mobile robot navigation system to avoid detected obstacles in a real environment.

\begin{tabular}{|c|c|c|c|c|c|}
\hline Detected & Avoided & Collisions & $\begin{array}{c}\text { Total } \\
\text { collision } \\
\text { free }\end{array}$ & $\begin{array}{c}\text { Collision-free } \\
\text { avoidance } \\
\text { accuracy }(\%)\end{array}$ & $\begin{array}{c}\text { Avoidance } \\
\text { accuracy with } \\
\text { collisions }(\%)\end{array}$ \\
\hline 111 & 80 & 29 & 51 & 72,07 & 45,95 \\
\hline
\end{tabular}

Table 2. Accuracy of the mobile robot navigation system

Previously presented results of accuracy of detection and avoidance of obstacles are derived from all previously mentioned system limitations, but also external influences and imperfections of the mobile robot drive system. Imperfections of the drive system of the mobile robot have proven to be another factor that partially affects accuracy, especially in cases where the robot needs to make longer movements to avoid an obstacle. There are also some increment estimation losses in incremental encoders, calculation time losses and data transfer losses between the robot and the laptop.

Losses caused by friction should also be taken into account. Respectively, friction losses due to the contact of the wheels with the ground (depends on current conditions and type of the ground) and friction losses caused when the rear wheel is inactive and "is dragging" on the ground. All these degradations cause a deviation so that the Robotino does not actually cross a distance of $1 \mathrm{~m}$, but this distance averages $0.915 \mathrm{~m}$ at a speed of $100 \mathrm{~mm} / \mathrm{s}$. Respectively, at a distance of $1 \mathrm{~m}$ the average deviation is $0.086 \mathrm{~m}$, and this can cause inaccurate and imprecise avoidance of obstacles at longer distances. 


\section{Conclusion}

This paper presents development and application of a mobile robot navigation control algorithm, which is realized with the application of artificial neural networks for sensor data fusion. This navigation system, based on the fusion of three infrared sensors and one vision sensor, was used to conduct an experiment for detecting and avoiding various types of obstacles indoors. The experiment was conducted in a real environment with real obstacles. Different types of obstacles were used in the experiment. Obstacles were detected in different environmental conditions, with different hardware and software limitations of the system and with different external influences. With all the above limitations and defined conditions, the experiment showed that the developed algorithm gives very good results (accuracy: 72.07\%), and the navigation system performs the tasks of detecting and avoiding most of the obstacles on which it was tested. Sensor fusion has proven to be a much better solution than the separate use of infrared sensors or vision sensors. Artificial neural networks have been proven to be a very good tool for application in systems where the input information is not always unambiguous and of the same character.

The disadvantages of the proposed navigation system are primarily the ability to move the robot only left, right and straight, without rotation, backward movement and combined movements (left-forward, right-forward, left-back, rightback). These movements cannot be performed without the use of a sensor that will cover area of $360^{\circ}$ around the robot. This was not the case in this experiment due to the application of only three front infrared sensors and a camera that is fixed and covers only a certain area in front of the robot. For these reasons, all the results of the application of the proposed algorithm should be analysed within the presented limitations in the hardware, software, sensor system and environment in which the experiment was performed.

The most important upgrade, to improve the system, is the use of additional infrared sensors and an omnidirectional camera that will have the ability to move (rotate) and thus cover a larger area around the robot. Future work may focus on the application of other methods of sensor fusion and data processing, which can be incorporated into the existing algorithm. Further research includes upgrading the algorithm with other types of sensors, such as ultrasound, laser scanners, 3D or thermal cameras, etc.

\section{References}

[1] B. Crnokić, (2016) "Development of the algorithm for control of mobile robot navigation with application of artificial neural networks," University of Mostar

[2] Y. Qingmei and S. Jianmin, (2008) "A data fusion method applied in an autonomous robot," 2008 27th Chinese Control Conf., pp. 361-364, Jul. doi: 10.1109/CHICC.2008.4605358.

[3] V. Andreev and V. Tarasova,(2019) "The mobile robot control for obstacle avoidance with an artificial neural network application,” Ann. DAAAM Proc. Int. DAAAM Symp., vol. 30, no. 1, pp. 724-732, 2019, doi: 10.2507/30th.daaam.proceedings.099.

[4] M. Kirola, D. Brezak, and D. Majetić, (2001) "Neural Network in the Autonomous Mobile Robot Path Tracking Control," in Proceedings of the 12th International DAAAM Symposium / Katalinic, Branko - Vienna : DAAAM International Vienna, 2001, pp. 223-224.

[5] Z.-G. H. Z.-G. Hou, M. T. M. Tan, M. M. Gupta, P. N. Nikiforuk, and N. Homma, (2005)“Neural network methods for the localization and navigation of mobile robots," Can. Conf. Electr. Comput. Eng. 2005., no. May, pp. 10571060

[6] M. T. An-Min Zou, Zeng-Guang Hou, (2005) "Support Vector Machines (SVM) for Color Image Segmentation with Applications to Mobile Robot Localization Problems," Int. Conf. Intell. Comput. ICIC 2005, vol. II, no. Springer Berlin Heidelberg, p. pp 443-452

[7] J. L. Crowley, F. Wallner, and B. Schiele, (1998.) "Position Estimation Using Principal Components of Range Data James L. Crowley, Frank Wallner and Bernt Schiele,” Robot. Autom. 1998., vol. 4., no. IEEE International, pp. 3121-3128,

[8] L. Yenilmez and H. Temeltas, (2006) "A new approach to map building by sensor data fusion: sequential principal component-SPC method," Int. J. Adv. Manuf. Technol., vol. 34, no. 1-2, pp. 168-178, Jun. 2006, doi: 10.1007/s00170-006-0578-3.

[9] L. Dong, (2010) "A Study on Multiple Information Fusion Technology in Obstacle Avoidance of Robots," Circuits,Communications Syst. (PACCS), 2010 Second Pacific-Asia Conf., vol. 1, pp. 171-174, 2010.

[10] F. U. Changhong, W. U. Shunxiang, L. U. O. Zhifeng, F. A. N. Xu, and M. Fanling, (2009) "Research and design of the differential autonomous mobile robot based on multi-sensor information fusion technology," Inf. Eng. Comput. Sci. 2009. ICIECS 2009. Int. Conf., pp. 1-4,

[11] I. E. T. Fujii, Y. Arai, H. Asama, (1998) "Multilayered reinforcement learning for complicated collision avoidance problems," Robot. Autom. 1998. Proceedings. 1998 IEEE Int. Conf., pp. 2186-2191

[12] G. G. Kim, H. H., Ha, Y. S., Jin, (2003) "A Study on the Environmental Map Building for a Mobile Robot Using Infrared Ranger-finder Sensors,” Intell. Robot. Syst. 2003. (IROS 2003)., vol. 1, pp. 711-716 
[13] M. Zou, A. , Hou, Z.G., Zhang, L., Tan, (2005) “A Neural Network-based Camera Calibration Method for Mobile Robot Localization Problems,” Proceeding Second Int. Symp. Neural Networks, vol. 13, pp. 277-284

[14] S. B. Thrun, (1993) "Exploration and Model Building in Mobile Robot Domains," Proc. IEEE Int. Conf. Neural Networks, vol. 1, pp. 175-180,

[15] H. M. Barberá, A. G. Skarmeta, and M. Z. Izquierdo, (2000) "Neural Networks for Sonar and Infrared Sensors Fusion," Proc. Third Int. Conf. Inf. Fusion, vol. 2, pp. 18.-25.

[16] J. Eduardo and C. Ortiz, (2012) "Visual Servoing for an Omnidirectional Mobile Robot Using the Neural network Multilayer Perceptron,” Eng. Appl. (WEA), 2012 Work., pp. 1-6

[17] O. Akinola, (2012) "The Development of Neural Network based Vision for an Autonomus Vehicle," Obafemi Awolowo University, Ile-Ife, Nigeria

[18] B. Crnokić and M. Grubišić, (2018) "Fusion Of Infrared Sensors And Camera For Mobile Robot Navigation System - Simulation Scenario," in Proceedings of 13th International Scientific Conference Novi Sad, Serbia, September 28 29, 2018, 2018, pp. 71.-75. 